\title{
Bremsstrahlung neutrinos from electron-electron scattering in a relativistic degenerate electron plasma
}

\author{
Prashanth Jaikumar*1, Charles Gale ${ }^{2}$, and Dany Page ${ }^{3}$ \\ ${ }^{1}$ Physics Division, Argonne National Laboratory, Argonne IL 60439, USA. \\ ${ }^{2}$ Department of Physics, McGill University, Montréal QC H3A 2T8, Canada. \\ ${ }^{3}$ Instituto de Astronomía, Universidad Nacional Autónoma de México, México D.F. 04510, Mexico.
}

We present a calculation of neutrino pair bremsstrahlung due to electron-electron scattering in a relativistic degenerate plasma of electrons. Proper treatment of the in-medium photon propagator, i.e., inclusion of Debye screening of the longitudinal part and Landau damping of the transverse part, leads to a neutrino emissivity which is several orders of magnitude larger than when Debye screening is imposed for the tranverse part. Our results show that this in-medium process can compete with other sources of neutrino radiation and can, in some cases, even be the dominant neutrino emission mechanism. We also discuss the natural extension to quark-quark bremsstrahlung in gapped and ungapped quark matter.

PACS: 26.60.+c, 95.30.Cq, 97.60.Jd

\section{INTRODUCTION}

For many astrophysical objects (e.g., supernovae or neutron stars) in which high densities and/or temperatures are attained, energy losses by neutrino emission are the driving force in their evolution [1-3]. The simplest process of $\beta$ and inverse $\beta$-decay of nucleons, the so-called direct Urca process, leads to prodigious energy losses [4] but is possible only in cases where free nucleons are present and, even in this case, can be kinematically forbidden. Similar direct Urca processes are also possible when hyperons [5] or quarks [6] are present and the associated emissivities dominate the cooling, but the existence of these exotic phases is subject to large uncertainties. In many cases, neutrinos can only be emitted through higher order processes in which many-body effects in the medium become important. A classic example is the plasmon decay process of neutrino pair emission where the very existence of plasmons is due to the medium's response to electromagnetic perturbations. Neutrino radiation via bremsstrahlung comprises a large subset of all neutrino emission processes where scattering of particles, through electromagnetic or strong interactions, allows transfer of energy-momentum accompanied by the emission of a $\mathrm{Z}^{0}$ boson which immediately decays into a $\nu-\bar{\nu}$ pair. Typical and well studied examples are, among others, the case of nucleon-nucleon, electron-ion and electronphonon scattering in the neutron star's core and crust (see, e.g., [7] for a recent review). However, neutrino pair bremsstrahlung emission in collisions of relativistic particles has received little attention and only a few attempts exist in the literature, e.g., [8]. In this paper, we will be concerned with the importance of the medium in the modulation of the scattering interaction of relativistic electrons through polarization effects, and its impact on the neutrino emissivity.

In a QED plasma, static electric fields are screened while magnetic fields suffer damping only at non-zero frequency. For scattering in the relativistic limit, when magnetic effects are important, soft photon exchange can lead to a divergence from the unscreened magnetic interaction. To avoid this divergence, in early works on transport coefficients in a QED plasma, Debye screening was imposed in both polarization channels of the photon propagator in the covariant gauge, even though it only applies to its longitudinal "electric" part. It was later duly noted $[9,10]$ that Landau damping of the transverse "magnetic" part can generate a finite result in these calculations. Landau damping instead of Debye screening of the magnetic interaction implies higher scattering rates and hence smaller thermal and electrical conductivities and shear viscosity [10]. Similarly, higher scattering rates should result in larger neutrino emissivities for bremsstrahlung processes, and this is the issue we address here by calculating the neutrino pair emission from electron-electron bremsstrahlung in a degenerate relativistic electron plasma. Our results confirm these heuristic arguments and show that erroneously assuming Debye screening of the magnetic interaction can underestimate the neutrino emissivity by several orders of magnitude, particularly at the low temperatures of relevance to a variety of astrophysical environments.

*jaikumar@phy.anl.gov 
This paper is organized as follows: in section II, we discuss the many-body effects that are relevant for the temperature and density region under consideration and which must be taken into account. Section III outlines the principal steps in the calculation of the neutrino bremsstrahlung emissivity from electron-electron collisions. We present a comparison of the results with other neutrino rates in three different contexts : from gapped and ungapped quark matter in strange stars in section IV, from hot matter in a black-hole accretion torus in section V, and finally in the more conventional medium of a neutron star, both in its crust and in its core, in section VI. Our conclusions and outlook are presented in section VII. Some numerical details are explained in the appendix.

\section{NEUTRINO BREMSSTRAHLUNG IN ELECTRON-ELECTRON COLLISIONS}

We are interested primarily in the effects of Landau damping on the bremsstrahlung rate since the screened case has already been considered in [8]. A unified treatment, with consistent inclusion of plasma effects, of neutrino bremsstrahlung in electron-ion and electron-phonon collisions and plasmon decay in the crust of neutron stars is described in [11]. Here, we derive the emissivity for bremsstrahlung in scattering of relativistic electrons, which is relevant to a host of astrophysical conditions, and the results are also extended to quark-quark bremsstrahlung, where the exchanged gluon is similarly dressed. Neutrino bremsstrahlung is computed from the set of tree-level Feynman graphs displayed in Fig. 1. Before performing the explicit computation, we discuss some many-body effects that can potentially be important for our temperature and (electron or quark) density regime.

a) Landau damping: In the electron plasma, the exchanged photon is dressed by interaction with the electrons. The longitudinal photon is Debye screened, while the transverse photon is Landau damped. At the one-loop level, an all-order resummation leads to a photon propagator that is modified from its "bare" expression as follows:

$$
D^{\mu \nu}(\omega, k)=\frac{P_{L}^{\mu \nu}}{K^{2}-\Pi_{L}}+\frac{P_{T}^{\mu \nu}}{K^{2}-\Pi_{T}}
$$

where $K^{2}=\omega^{2}-k^{2}$ and $P_{L}^{\mu \nu}, P_{T}^{\mu \nu}$ are the longitudinal and transverse projection operators in [12]. The longitudinal and transverse self-energies $\Pi_{L}$ and $\Pi_{T}$ are in general complex functions of $\omega$ and $k$. These self-energies can be obtained in the hard dense loop approximation ${ }^{1}$. Since the exchanged photon also has small energy, we will use approximate forms [12] in the nearly static limit $\omega / k \ll 1$ :

$$
\Pi_{L}=m_{D}^{2} \quad \text { and } \quad \Pi_{T}=m_{M}^{2}=i\left(\frac{\pi}{4}\right) \frac{\omega}{k} m_{D}^{2}
$$

For degenerate electrons, characterized by a chemical potential $\mu_{e}$,

$$
m_{D}^{2}=e^{2} \mu_{e}^{2} / 3
$$

but for the sake of generality, we use $m_{D}$ to denote the phase-dependent screening mass. Its explicit expression is to be understood by the particular case under consideration.

b) Vertex renormalization: Neutrinos are emitted with average energies that are thermal, and in the degenerate regime $T / \mu_{e} \ll 1$. This implies that their wavenumber $k_{\nu}$ is such that $k_{\nu}^{2} / m_{D}^{2} \ll 1$. Physically, this means that at sufficiently low temperatures, and large electron densities, the neutrino's wavelength is comparable to or exceeds the Debye charge radius. In this case, as shown by Leinson [11], the effective weak charge seen by the neutrino is renormalized. The correction factor due to this effect can be absorbed simply into "effective" electroweak couplings, and we take this into account in our calculations.

c) Non-Fermi liquid effects: In normal quark matter with $N_{f}$ quark flavors at a common chemical potential $\mu$, the absence of screening for the chromomagnetic gluon leads to a breakdown of Fermi liquid behaviour for quarks at a scale $T \sim m \exp \left(-9 \pi / 4 \alpha_{c}\right)$ [13] where $m^{2}=N_{f} \alpha_{c} \mu^{2} / \pi$ is the electric screening mass, with $\alpha_{c}=g^{2} / 4 \pi$ where $g$ is the QCD coupling constant. The neutrino emission rate for the quark direct urca process is consequently enhanced [14]. This effect applies also to the electron plasma, but we ignore it here since the smallness of the QED coupling constant implies that this effect sets in only at exponentially small temperatures.

\footnotetext{
${ }^{1}$ We are ignoring the smaller contribution from finite temperature effects (hard thermal loops) since they are small for a degenerate QED plasma.
} 


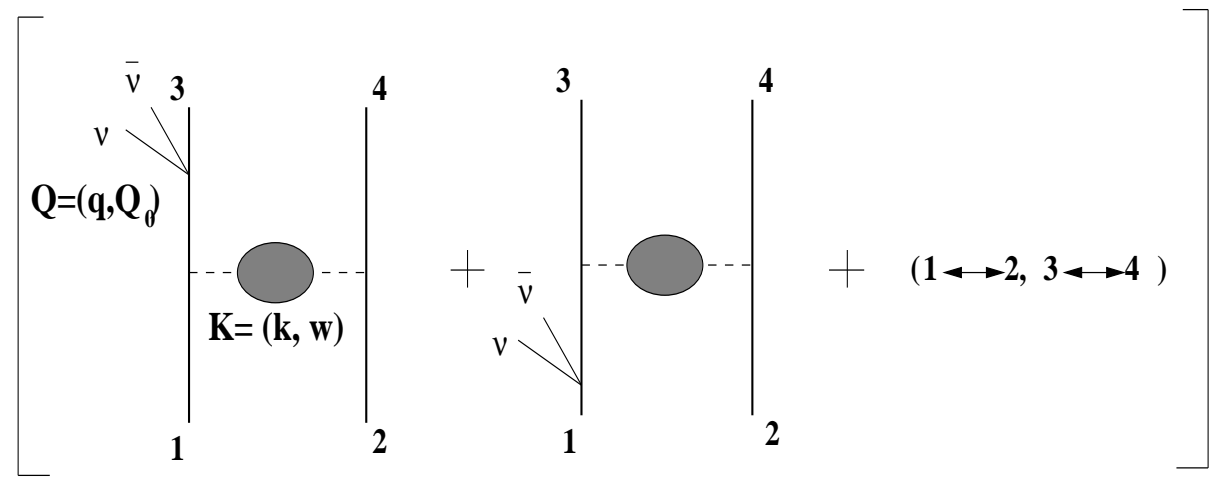

$+(3 \longleftrightarrow 4)$

FIG. 1. The Feynman diagrams for neutrino bremsstrahlung in electron-electron scattering. The first 4 diagrams, enclosed within square brackets, are the "direct" contributions while the interchange $(3 \leftrightarrow 4)$ generates the "exchange" diagrams. The blob represents the one-loop resummed photon propagator (see text for details).

d) LPM effect: Suppression of soft radiation due to multiple scatterings between electrons (the LandauPomeranchuk-Migdal or LPM effect) becomes important when the formation time $t_{f}$ of the neutrino wave-packet is comparable to the mean collision time $t_{c}$ between electrons. The ratio $t_{f} / t_{c}$, for a degenerate plasma $\left(T \ll \mu_{e}\right)$ behaves approximately as $\sqrt{\alpha} T / \mu_{e}$ if only screening is considered, and as $\alpha$ when damping is taken into account $(\alpha$ is the fine structure constant). In either case, the ratio is much smaller than 1 , so that an inclusion of the LPM effect would make only a $1 \%$ correction to our results. The large degeneracy at the Fermi surface implies that the mean time between electron-electron collisions is much greater than the formation time of the neutrino wave-packet.

\section{NEUTRINO EMISSIVITY FROM THE BREMSSTRAHLUNG PROCESS}

The neutrino emissivity from the bremsstrahlung process is

$$
\begin{aligned}
Q_{\mathrm{ee}-\mathrm{Br}}=\frac{2 \pi}{\hbar} & {\left[\prod_{i=1}^{4} \int \frac{d^{3} p_{i}}{(2 \pi)^{3} 2 E_{i}}\right] \int \frac{d^{3} q_{1}}{(2 \pi)^{3} 2 \omega_{1}} \frac{d^{3} q_{2}}{(2 \pi)^{3} 2 \omega_{2}} Q_{0} \sum_{\text {spin }} \frac{|M|^{2}}{s} } \\
& \times n_{F}\left(E_{1}\right) n_{F}\left(E_{2}\right) \tilde{n}_{F}\left(E_{3}\right) \tilde{n}_{F}\left(E_{4}\right)(2 \pi)^{3} \delta^{3}\left(\mathbf{P}_{\mathbf{f}}-\mathbf{P}_{\mathbf{i}}\right) \delta\left(E_{\mathbf{f}}-E_{\mathbf{i}}\right) .
\end{aligned}
$$

The subscripts $i=1$ to 4 on $p$ refer to electrons (1,2 label the incoming states and 3,4 the outgoing states), while $\mathbf{i}, \mathbf{f}$ refer to total initial and final three-momentum/energy accordingly. $Q_{1}=\left(\omega_{1}, q_{1}\right), Q_{2}=\left(\omega_{2}, q_{2}\right)$ are respectively the neutrino and anti-neutrino four-momenta with $Q=\left(Q_{0}, q\right)=\left(\omega_{1}+\omega_{2}, q_{1}+q_{2}\right)$. The symmetry factor $s=2$. The phase space for electrons is convolved with the appropriate Fermi distribution functions $n_{F}\left(E_{i}\right)=1 /\left(\mathrm{e}^{\left(E_{i}-\mu_{e}\right) / T}+1\right)$ (in $k_{B}=1$ units) and $\tilde{n}_{F}=1-n_{F}$, respectively. The matrix element $M$ is obtained by the application of Feynman rules to the diagrams shown in Fig. 1. Since we work in the degenerate regime, the emissivity is dominated by contributions from low energy neutrinos (typically of order the temperature $T$, where $T \ll \mu_{e}$ ) and Low's theorem [15] may be used to simplify the calculation of $Q_{\mathrm{ee}-\mathrm{Br}}$. As a consequence, the propagator of the internal fermion line can be approximated as

$$
S_{e}(p \pm Q)= \pm \frac{1}{Q_{0}} \quad .
$$

A further approximation can be made by noting that interference between the direct and exchange diagrams is strongly suppressed relative to their squares taken individually, and hence may be neglected [8]. Then, in the low-energy limit, 
the emission from the exchange diagrams simply doubles the overall result for the emissivity from the direct diagrams. Since there is a symmetry factor of $s=2$ in the denominator of the emissivity expression eqn. (4), we may ignore the identity of the particles altogether and evaluate the emissivity from the direct diagrams alone (see caption Fig. 1).

Aside from the equation for the dressed photon propagator (eqn.(1)), we also require the weak interaction Hamiltonian (in the 4-Fermi form) given by

$$
\begin{aligned}
H_{4 f} & =\frac{G_{F}}{2 \sqrt{2}} \Gamma_{\lambda} \bar{\psi}_{\nu} \gamma^{\lambda}\left(1-\gamma_{5}\right) \psi_{\nu} \\
\Gamma_{\lambda} & =\bar{\psi}_{e} \gamma_{\lambda}\left(c_{V}-\gamma_{5} c_{A}\right) \psi_{e}
\end{aligned}
$$

The weak vector and axial-vector couplings are

$$
\begin{aligned}
c_{V}^{e} & =1+4 \sin ^{2} \theta_{W}, \quad c_{A}^{e}=1 . \\
c_{V}^{\mu, \tau} & =-1+4 \sin ^{2} \theta_{W}, \quad c_{A}^{\mu, \tau}=-1 .
\end{aligned}
$$

where the Weinberg angle $\sin ^{2} \theta_{W}=0.231$. Neutrino-pairs of all three flavors $(e, \mu, \tau)$ contribute incoherently to the emissivity, so that we may define a "total" squared coupling constant $c_{+}^{2}=\sum_{\nu}\left(\left(c_{V}^{\nu}\right)^{2}+\left(c_{A}^{\nu}\right)^{2}\right)=6.696$. The vertex renormalization effect discussed in the previous section introduces a suppression factor $s=0.449$. This implies a total "effective" squared coupling constant $c_{\text {eff }}^{2}=3.006$. We will use the symbols $c_{V}$ and $c_{A}$ for the following calculation, and substitute the effective coupling constant only at the end. Evaluating the spin-summed squared matrix element with the low-energy approximations for neutrinos, and utilizing symmetries between the electron "labels" $(1,2,3,4)$, we obtain (neglecting terms of $\mathcal{O}\left(m_{e}^{2} / \mu_{e}^{2}\right)$ )

$$
\begin{aligned}
\frac{|M|^{2}}{s} & =\frac{256 e^{4} G_{F}^{2}}{Q_{0}^{2}} L\left(Q_{1}, Q_{2}\right)^{\rho \lambda} D^{\alpha \mu} D^{\beta \nu} p_{2 \alpha} p_{2 \beta} \times\left[i c_{V} c_{A} \epsilon_{\nu \lambda}^{\gamma \delta}(T 1)_{\gamma \delta \mu \rho}+\left(c_{V}^{2}+c_{A}^{2}\right)(T 2)_{\nu \lambda \mu \rho}\right], \\
(T 1)_{\gamma \delta \mu \rho} & =\left[\left\{g_{\delta \rho}\left(p_{1 \mu} p_{3 \gamma}+1 \leftrightarrow 3\right)+g_{\gamma \mu}\left(p_{1 \rho} p_{3 \delta}+1 \leftrightarrow 3\right)+p_{1} \cdot p_{3}\left(g_{\gamma \rho} g_{\mu \delta}\right)\right\}-(\gamma \leftrightarrow \delta)\right], \\
L\left(Q_{1}, Q_{2}\right)^{\rho \lambda} & =8\left(Q_{1}^{\lambda} Q_{2}^{\rho}+Q_{1}^{\rho} Q_{2}^{\lambda}-g^{\lambda \rho} Q_{1} \cdot Q_{2}-i \epsilon^{\mu^{\prime} \nu^{\prime} \lambda \rho} Q_{1 \mu^{\prime}} Q_{2 \nu^{\prime}}\right) .
\end{aligned}
$$

All vectors appearing in the above equation are 4 -vectors, and $T 2$ is obtained from $T 1$ with the interchange of indices $(\delta \leftrightarrow \lambda, \gamma \leftrightarrow \nu)$. The neutrino phase space integrals are easily performed by introducing a delta function as

$$
1=\int d^{4} Q \delta^{4}\left(Q-Q_{1}-Q_{2}\right)
$$

and then using Lenard's identity

$$
N_{\alpha \beta}=\int \frac{d^{3} Q_{1}}{2 \omega_{1}} \frac{d^{3} Q_{2}}{2 \omega_{2}} Q_{1 \alpha} Q_{2 \beta} \delta^{4}\left(Q-Q_{1}-Q_{2}\right)=\frac{\pi}{24}\left(Q^{2} g_{\alpha \beta}+2 Q_{\alpha} Q_{\beta}\right) \Theta\left(Q_{0}\right) \Theta\left(Q_{0}^{2}-\mathbf{q}^{2}\right)
$$

Before performing further contractions, we choose to retain only the transverse part of the photon propagator in eqn.(8). We will justify this a posteriori when the contribution from Landau damping is found to be much larger than naive screening [8]. We focus on this effect, which is expected to be important due to the exchange of soft photons. The remaining tensor contractions in eqn.(8) finally yield

$$
\begin{aligned}
Q_{\mathrm{ee}-\mathrm{Br}}= & A \int d Q_{0} Q_{0}^{4} P \\
A= & \left(\frac{16}{45}\right) \frac{1}{(2 \pi)^{12}} \frac{e^{4} G_{F}^{2}\left(c_{V}^{2}+c_{A}^{2}\right)}{\hbar p_{F_{e}}^{4}} \\
P= & {\left[\int \prod_{i=1}^{4} d^{3} p_{i}\right] n_{F}\left(E_{1}\right) n_{F}\left(E_{2}\right) \tilde{n}_{F}\left(E_{3}\right) \tilde{n}_{F}\left(E_{4}\right) \delta^{3}\left(\mathbf{P}_{\mathbf{f}}-\mathbf{P}_{\mathbf{i}}\right) \delta\left(Q_{0}+E_{\mathbf{f}}-E_{\mathbf{i}}\right) } \\
& \times \frac{1}{\left|K^{2}-m_{M}^{2}\right|^{2}} \times\left\{\left|\mathbf{p}_{2} \times \hat{\mathbf{k}}\right|^{2}\left(13 E_{1} E_{3}-\mathbf{p}_{1} \cdot \mathbf{p}_{3}\right)\right\}
\end{aligned}
$$

where boldface quantities denote 3 -vectors. The momentum integrals over $d^{3} p_{2}, d^{3} p_{3}$ are performed first using the techniques described in [10]. Since the electron mass $m_{e} \ll p_{F_{e}}$, the electron Fermi momentum, the electron energies $E_{i}$ may be traded for the momentum magnitudes $p_{i}$ at the Fermi surface. Then $P$ in eqn.(15) becomes 


$$
\begin{aligned}
P= & 24 \pi^{2} \int_{-\infty}^{\infty} d \omega \int \frac{d^{3} k}{k^{2}\left|K^{2}-m_{M}^{2}\right|^{2}} \Theta(k-|\omega|) \int_{\frac{k+\omega}{2}}^{\infty} d p_{4} p_{4}\left(p_{4}-\omega\right)^{3} \sin ^{2} \theta_{0} \tilde{n}_{F}\left(p_{4}\right) n_{F}\left(p_{4}-\omega\right) \\
& \times\left\{\theta(\omega) \theta\left(k-\left(\omega+Q_{0}\right)\right)+\theta(-\omega)\left[\theta\left(Q_{0}-|\omega|\right) \theta\left(k-\left(Q_{0}-|\omega|\right)\right)+\theta\left(|\omega|-Q_{0}\right) \theta\left(k-\left(|\omega|-Q_{0}\right)\right)\right]\right\} \\
& \times \int_{\frac{k+\omega+Q_{0}}{2}}^{\infty} d p_{1} p_{1}\left(p_{1}-\omega-Q_{0}\right)^{3} n_{F}\left(p_{1}\right) \tilde{n}_{F}\left(p_{1}-\omega-Q_{0}\right), \\
\cos \theta_{0}= & \left(\frac{k^{2}-\omega^{2}+2 p_{4} \omega}{2 p_{4} k}\right) .
\end{aligned}
$$

Next, the $d p_{1}, d p_{4}$ integrals are performed, which fixes the limits on the $d k$ and $d \omega$ integrations. At this stage, it is convenient to perform the $d^{3} k$ integral, noting that $\sin ^{2} \theta_{0}$ is a function of $\omega, k$. Explicitly, this integral is of the form

$$
I_{k}=\int_{l_{1}}^{2 \mu_{e}} d k \frac{\sin ^{2} \theta_{0}}{\left(\omega^{2}-k^{2}\right)^{2}+m_{M}^{4}}
$$

where $l_{1} \sim T$ is the lower integration limit as determined by the $\Theta$-functions involving $k$. The evaluation of this integral is detailed in appendix A. The result is

$$
I_{k} \approx \frac{4}{3 \pi m_{D}^{2} \omega}\left[\frac{\pi}{2}-\tan ^{-1}\left(\frac{l_{1}^{3}}{\frac{\pi}{4} m_{D}^{2} \omega}\right)\right] .
$$

We restrict our temperature and density range such that this approximation makes only a $10 \%$ error in the final analytic expression for the emissivity, which is acceptable. Using this approximation, $P$ reduces to

$$
\begin{aligned}
P & =\frac{128 \pi^{2}}{m_{D}^{2}} \mu_{e}^{8} T^{2} N(\alpha), \\
N(\alpha) & =\int_{0}^{\infty} d \bar{\Omega} \bar{\Omega}^{4} \mathrm{e}^{-\bar{\Omega}} I(\bar{\Omega}) ; \quad \bar{\Omega}=\frac{Q_{0}}{2 T}, \quad \alpha=\frac{m_{D}}{2 T} .
\end{aligned}
$$

where $I(\bar{\Omega})$ is a smooth function of $\bar{\Omega}$ obtained upon integration over $\omega$ (see Appendix B for details). Substituting the resultant expression for $P$ from eqn.(20) in eqn.(13), we are left with an overall integral over $Q_{0}$, which is numerically tractable. The final result for the neutrino emissivity reads (we have chosen natural units $\hbar=c=1$; a factor of $\hbar^{10} c^{9}$ should be inserted in the denominator to recover "standard" units)

$$
Q_{\mathrm{ee}-\mathrm{Br}}=\left(\frac{16}{45 \pi^{10}}\right) \frac{e^{4} G_{F}^{2} c_{\mathrm{eff}}^{2} T^{7} \mu_{e}^{4}}{m_{D}^{2}} N(\alpha)
$$

Substituting the values of the physical constants, and defining $T_{9}$ as the temperature in units of $10^{9} \mathrm{~K}, \mu_{10}$ as the electron chemical potential in units of $10 \mathrm{MeV}$, we obtain

$$
Q_{\mathrm{ee}-\mathrm{Br}}=4.6 \times 10^{16} T_{9}^{7} \mu_{10}^{2} \tilde{N}(\alpha) \mathrm{erg}^{-3} \mathrm{~cm}^{-3} \mathrm{~s}^{-1} .
$$

where we also introduced a normalized $\tilde{N}(\alpha)=N(\alpha) / 212$ such that $\tilde{N}(\alpha) \leq 1$ (See Figure 2). The emissivity is plotted as a function of temperature for an electron chemical potential $\mu_{e}=30 \mathrm{MeV}$ in Fig.3. For comparison, the emissivity as computed from eqn.(51) of ref. [8], which assumed Debye screening of both the longitudinal and transverse parts of the photon propagator, is also shown. The inclusion of Landau damping has modified the temperature and density dependence of the emissivity from the case of pure screening. In our case, the emissivity scales as $\mu_{e}^{2} T^{7}$ while in the screened case, it behaves as $\mu_{e} T^{8}$. Note that in our result, the quantity $N(\alpha)$ is also temperature dependent, so that a slight curvature in the log-plot of emissivity versus temperature is to be expected. In case of [8], a similar numerical integral appears but is temperature-independent and their result yields a straight line. The $T_{9}^{7}$ dependence implies a slower fall-off of the emissivity with decreasing temperature, reflecting the increased importance of soft photon exchange at lower temperatures. This is due to the fact that electrons have smaller thermal energies, and cannot scatter far from the Fermi surface. The soft exchanges are not screened, and this leads to the enhancement of the emissivity, when compared to previous results which did not incorporate the Landau damping. At high temperatures $\left(T_{9} \gg 10\right.$ and beyond), the electrons start to become non-degenerate, and our approximations would break down. Nevertheless, the convergence of our result to that of ref. [8] with increasing temperature is expected since both must approach the emissivity computed in the non-degenerate case [16]. 


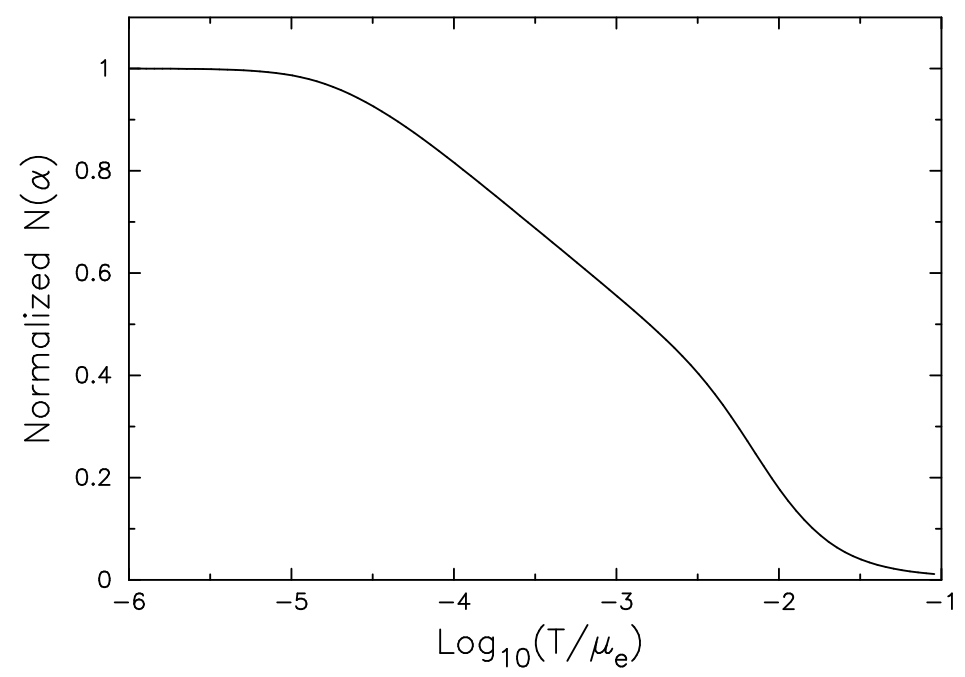

FIG. 2. The normalized $\tilde{N}(\alpha) \equiv N(\alpha) / 212$ from eqn.(21)

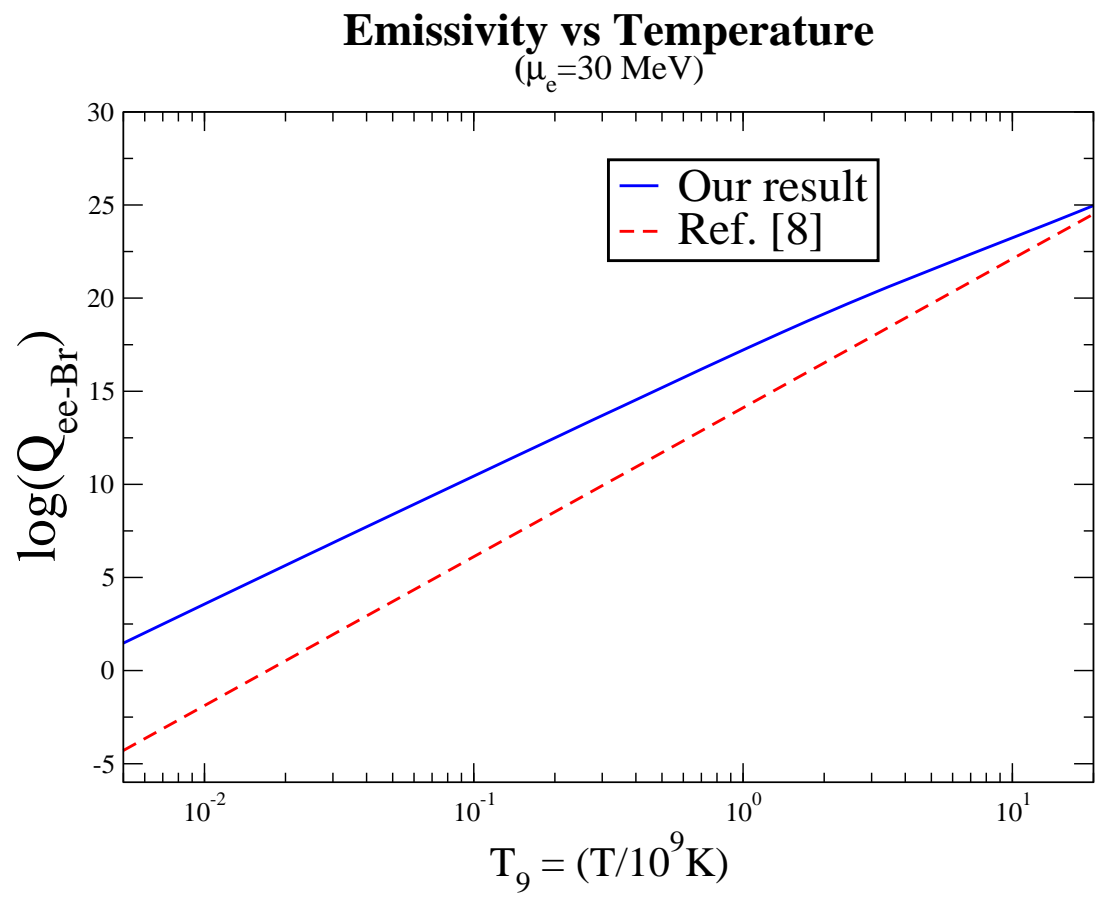

FIG. 3. The neutrino emissivity (on a log scale) from eqn.(23) of this paper and eqn.(51) of [8]. The chemical potential is fixed at $\mu_{e}=30 \mathrm{MeV}$.

There are other sources of neutrino emission from a QED plasma, such as the plasmon process [17,18], where an in-medium photon dressed by the electron plasma can decay into $\nu \bar{\nu}$ pairs, and the plasma photo-neutrino process [19] $e^{ \pm}+\gamma \rightarrow e^{ \pm}+\nu \bar{\nu}$. Formally, the plasmon process can interfere with $\nu \bar{\nu}$ bremsstrahlung when the exchanged photon in electron-electron collisions is almost on-shell. This is because the lifetime of such a photon is very long and it can be regarded as a valid in-medium excitation just like a plasmon. However, these two contributions can be disentangled when the temperature and density are such that only one of them dominates [20]. Studies of the plasmon process have shown that for a degenerate electron plasma at low temperatures such that $T \ll \omega_{P}$, where $\omega_{P}$ is the plasma frequency, the total emissivity acquires an exponential factor $\mathrm{e}^{-\omega_{P} / T}$. For example, the emissivity from the decay of 
the transverse mode (photon) is ${ }^{2}[18]$

$$
Q_{T} \simeq \frac{c_{V}^{2} G_{F}^{2}}{48 \pi^{4} \alpha} \sqrt{\frac{\pi}{2}} \omega_{P}^{15 / 2} T^{3 / 2} \mathrm{e}^{-\omega_{P} / T}
$$

The plasma frequency $\omega_{P}$ for a relativistic degenerate plasma is a function of the electron chemical potential

$$
\omega_{P}^{2} \cong \frac{4 \alpha}{3 \pi} \mu_{e}^{2}\left(1+\frac{\pi^{2} T^{2}}{3 \mu_{e}^{2}}\right)
$$

For $\omega_{P} \sim \mathcal{O}(\mathrm{MeV})$ and for our temperature regime $(T \leq 1 \mathrm{MeV})$, the plasmon decay rate and the plasma photoneutrino rate are strongly suppressed, while the bremsstrahlung rate is not. In the following sections, we apply our main result, eqn.(23), to explore and compare neutrino rates in a variety of astrophysical environments.

\section{COMPARISON TO NEUTRINO RATES IN STRANGE STARS}

Self-bound quark matter made of up, down and strange quarks can exist at high density, as evidenced by model calculations [21]. An entire star made of this phase is known as a strange star. Having obtained a modified inmedium neutrino radiation rate, we would like to identify the physical conditions under which neutrino emission from relativistic electron-electron scattering, and quark-quark scattering, can affect strange star cooling.

\section{A. The strange star surface}

In case the star's surface is not covered by baryonic matter, it has a bare quark surface covered by a thin layer of degenerate electrons [22], termed the electrosphere. It has recently been suggested that the bare quark surface is not as sharp as previously thought but may rather consist of a crust of quark nuggets embedded in a degenerate electron gas [23]. These strange nuggets have larger electric charge than nuclei in a nuclear crust, but are very sparsely distributed, so that neutrino luminosity from electron-nugget bremsstrahlung will still be less than that from electron-electron bremsstrahlung, as calculated in this paper. Another intriguing scenario develops if the underlying quark matter is in a gapped phase, since the neutrino emissivity from quarks is severely attenuated at temperatures $T \ll T_{c}$ where $T_{c}$ is the critical temperature for pairing. The specific heat is similarly affected if electrons are not admixed with quark matter (as is the case with the color-flavor-locked or CFL phase [24]). Despite the enforced charge neutrality of the CFL phase in bulk, surface effects still lead to the formation of a degenerate electron plasma at the star's surface [25] and a possible nugget envelope along the lines of [23] may be present. Neutrinos from electron-electron bremsstrahlung in the envelope will then be the dominant energy sink for a strange star composed of stable CFL matter all the way up to its surface. In addition, the superfluid nature of the CFL phase implies a large thermal conductivity [26], so that neutrino cooling is determined by the neutrino emission properties of the surface [27]. Below, we examine the dominant sources for neutrino emission in the bulk of the strange star, since the true phase of quark matter at supra-nuclear density remains as yet unknown, and make a comparison to the surface or envelope emission rates.

\section{B. Ungapped quark matter}

If quarks of all flavors are ungapped, the direct urca process (weak equilibrium between $d$ and $u$ quarks) is dominant, with [28]

$$
Q_{\text {durca }}=8.8 \times 10^{26} \alpha_{c}\left(\frac{n_{B}}{n_{0}}\right) Y_{e}^{1 / 3} T_{9}^{6} \operatorname{erg~cm}^{-3} \mathrm{~s}^{-1}
$$

\footnotetext{
${ }^{2}$ The emissivity can be decomposed into longitudinal and transverse parts for the vector channel, and an axial channel. They are all exponentially suppressed as $\exp \left(-\omega_{P} / T\right)$ though the pre-factors are different [18].
} 
With typical values $\alpha_{c}=0.1$, baryon density $n_{B}=2 n_{0}\left(n_{0}=0.16 \mathrm{fm}^{-3}\right.$ is the saturation density of nuclear matter $)$ and electron fraction $Y_{e}=10^{-5}$, a comparison of eqn.(26) with eqn.(23) shows that the former dominates by more than 8 orders of magnitude for the entire temperature range under consideration. With the inclusion of the non-Fermi liquid effects in ungapped quark matter, the emissivity is [14]

$$
Q=Q_{\text {durca }}\left[1+0.014 \ln \left(\frac{235.85}{T_{9}}\left(\frac{n_{B}}{n_{0}}\right)^{1 / 3}\right)\right]^{2} \operatorname{erg~cm}^{-3} \mathrm{~s}^{-1} .
$$

Other neutrino emission processes from ungapped quark matter, such as from quark-quark scattering, also exceed the rate computed here. Therefore, the presence of ungapped quark matter below the surface obviates a consideration of neutrino emission from the surface, at least as regards the cooling behaviour of the star.

\section{Gapped quark matter}

In the CFL phase, all 9 quarks are gapped, and the expectation is that neutrino emission processes involving free quarks are suppressed by the energy cost to be paid in exciting them above the gap $\Delta \gg T$. This is not the complete story, however, since the CFL phase supports Goldstone modes ( 8 massive and 1 massless) that require much less energy to excite. Importantly, they can all couple to neutrinos, and contribute to the emissivity. Among them, the most important processes are the electroweak decay of the kaon, and of the exactly massless $H$ boson through a momentum dependent coupling to neutrinos [29]. The latter has a very weak temperature dependence [27] and can be effectively ignored until very late times in the star's history. A comparison of the neutrino rate from kaon decay (eqn.(30) in ref. [27]) with eqn(23) is shown in Fig.4, revealing that surface neutrino emission from the electrosphere starts to dominate for temperatures less than $8 \times 10^{8} \mathrm{~K}$ or so. The reason for the sharp fall-off of the neutrino emissivity in the CFL phase is the Boltzmann factor of $\exp \left(-m_{G B} / T\right)$ where $m_{G B}$, the mass of the Goldstone boson is typically $10 \mathrm{MeV}$.

\section{Emissivity vs Temperature}

$\left(\mu_{\mathrm{q}}=500 \mathrm{MeV}, \mu_{\mathrm{e}}=30 \mathrm{MeV}\right)$

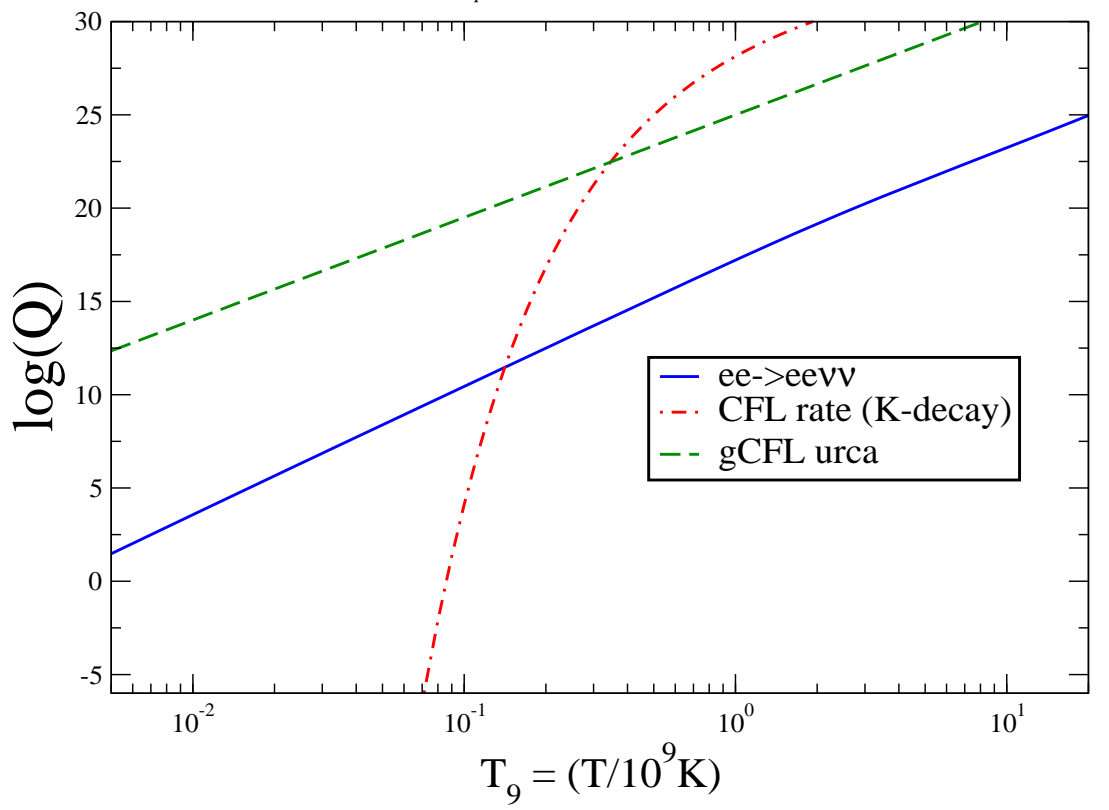

FIG. 4. Comparison of neutrino emissivity (on a log scale) from eqn.(23)(solid) with eqn.(30) of ref. [27](dashed), and eqn.(31) of ref. [36](dash-dotted). The quark chemical potential is fixed at $\mu_{q}=500 \mathrm{MeV}$ and the electron chemical potential at $\mu_{e}=30 \mathrm{MeV}$.

Stresses placed on the CFL phase by virtue of a finite strange quark mass can trigger the formation of new ground states. For example, the lightest massive boson can Bose-condense, leading to neutral kaon condensation (the CFLK 
phase) [30-32]. While the decreasing mass can initially increase the emissivity from the decay $K^{0} \rightarrow \nu \bar{\nu}^{3}$, phase space considerations eventually shut off neutrino emission. The dominant rates come from processes such as $K^{+} \rightarrow e^{+} \nu_{e}$ (and the analog for the $K^{-}$) and $K^{+} e^{-} \rightarrow \nu_{e}$, which can occur due to the dispersion relation turning space-like beyond a certain momentum. The associated neutrino rates are enhanced over the CFL rates, but still suffer from exponential suppression due to Boltzmann factors, and cannot compete with the surface emission when the temperature drops below $0.1 \mathrm{MeV}$ or so.

The CFL and CFLK ${ }^{0}$ phases are the likely ground state of dense quark matter only at extremely high quark chemical potential. At densities relevant to neutron stars, with $\mu_{q} \sim 500 \mathrm{MeV}$ or less, and with the physical requirements of charge and color neutrality, the pairing pattern can be quite complex involving phases with gapless modes for certain quark quasiparticles [33-35]. This opens up the possibility of a direct urca process that is not exponentially penalized even though the gap is non-zero, and the neutrino emissivity from gapless CFL matter (gCFL) was calculated in [36]. To facilitate a comparison of the rates, we choose (as in [36]), $\mu_{q}=500 \mathrm{MeV}, m_{s}^{2} / \mu_{q}=50$, and a gap $\Delta=25$ $\mathrm{MeV}$. From Fig.4, it is clear that this is a powerful channel for neutrino emission at all temperatures relevant to the long-term cooling of the star. It is a sensitive function of $m_{s}^{2} / \mu$, but the values used here are likely close to those in nature. Its effect on overall cooling only shows up in the photon-dominated cooling epoch however, due to the large specific heat of the gapless phase [36].

Several other color superconducting phases at intermediate densities relevant to neutron stars have been considered in the literature [37-39] which we do not address individually here. The question as to which is the preferred stable state of quark matter at intermediate densities and physical strange quark mass remains an open one at this time. We may mention here that a crystalline (LOFF) phase may exist at large values of the strange quark mass [40,41] and the neutrino emission from such a phase remains to be computed within the framework of the low-energy effective Lagrangian for this phase [42].

The enhancement of neutrino emission due to the inclusion of soft exchanges in electron-electron collisions can also be extended to quark-quark collisions, though the issue is much more subtle. Neutrino bremsstrahlung rates from quark-quark collisions have been previously computed in [28,43,44], although without damping in the transverse channel taken into account. A treatment of quark-quark scattering and transport properties in a degenerate quarkgluon plasma including this effect was undertaken in [10]. Having performed an explicit computation for electronelectron scattering, we may expect a simple extension to neutrino bremsstrahlung in quark-quark scatterings, and for different quark phases. For ungapped quark matter with chemical potential $\mu$ for a particular flavor, the emissivity from eqn.(22) applies to scattering between like-flavor quarks, with an extra factor of $4 / 3$ from color-counting and averaging, and with the replacements $e \rightarrow g$ for the coupling constant, $\mu_{e} \rightarrow \mu_{q}, c_{\mathrm{eff}}^{2} \rightarrow c_{q}^{2}$. The weak couplings for quarks $c_{q}$ are listed in Table 1 of [45]. The Debye mass is $m_{D}^{2}=\alpha_{c} \mu_{q}^{2} / \pi$. However, as pointed out in section II, at energies (temperatures) $\omega \sim m \mathrm{e}^{-9 \pi / 4 \alpha_{s}} \sim 10 \mathrm{~s}$ of $\mathrm{keVs}$, non-Fermi liquid effects can render a perturbative analysis invalid, leading to logarithmic enhancements in the quark self-energy and the neutrino rate in ungapped quark matter $[13,14]$. Already for $T_{9}<0.1$, non-Fermi liquid effects are expected to set in. Since we integrate over all exchange energies, a more accurate evaluation of bremsstrahlung neutrino rates should include the non-Fermi liquid effects. In this respect, the neutrino rate computed here will be somewhat modified for quark matter, but only very slightly so for a QED plasma. Further complications arise if quark matter is superconducting. For gapped quark matter, such enhancements as discussed here need only be considered when an ungapped quark species exists, and interacts via a gluon whose Meissner mass (but not the Debye mass) vanishes. In the CFL phase, all quarks are gapped, and all transverse gluons acquire a Meissner mass [46]. ${ }^{4}$ In the gapless phase, the issue of Meissner masses is far from clear [48-50]. However, a residual $U(1)$ symmetry in this phase implies that for a condensate that is neutral under the associated conserved charge (denoted $\tilde{Q})$, the Meissner mass vanishes. Since some quark species carry $\tilde{Q}$ charge however, the gCFL phase is not a $\tilde{Q}$ insulator, and the $\tilde{Q}$ photon (like the other gauge bosons in this phase) is likely screened. Finally, in the LOFF phase ${ }^{5}$, whose appearance may be linked to the chromomagnetic

\footnotetext{
${ }^{3}$ At first sight, such a process violates helicity conservation, but the presence of a preferred frame implies that the decay is indeed allowed in a frame moving with respect to the medium [27].

${ }^{4}$ The two-flavor superconductor, i.e, the 2SC phase admits ungapped quarks and unscreened gluons, but is energetically disfavored as a homogeneous phase in most studies [39,47].

${ }^{5}$ An alternate phase that can arise at intermediate density is the Overhauser phase, which like the LOFF phase, is anisotropic; however it seems not to be favored energetically unless the number of colors is large [51] or the number of space dimensions is reduced [52].
} 
instability of the gapless phases, the 5 gluons corresponding to the unbroken $S U(2)_{c}$ group acquire Debye masses due to the existence of blocking regions on the Fermi sphere, while the Meissner masses vanish [53]. Since $u$ and $d$ quarks of one color ("blue") as well as strange quarks of all colors (presumably too heavy to participate in pairing) remain unpaired, unscreened gluon exchange among them can lead to enhanced neutrino bremsstrahlung emissivities, although the dominant neutrino emission process would be the modified urca among ungapped light quarks (eg. $\left.d+d \rightarrow d+u+e^{-}+\bar{\nu}_{e}\right)^{6}$.

\section{COMPARISON TO NEUTRINO RATES IN BLACK-HOLE ACCRETION TORI}

Extreme conditions in which neutrino emission is crucial for the system evolution are also found in accretion tori around black-holes. Such tori are essential ingredients of most Gamma-Ray Burst models, either in the collapsar scenario for long bursts [54] or in the neutron star-neutron star or neutron star-black hole merger scenarios for short bursts (see, e.g., [55] and [56]). With central densities above $10^{12} \mathrm{~g} \mathrm{~cm}^{-3}$ and temperatures in the range of 1 to $10 \mathrm{MeV}$, nucleons form an almost ideal gas while electrons are mildly degenerate. Considering, for illustration, comparable fractions of neutrons and protons, one has for the electron chemical potential $\mu \sim 10 \rho_{10}^{1 / 3} \mathrm{MeV}$. In the outer part of the torus, electrons are non-degenerate and thus positrons are present.

A reliable comparison of the various neutrino emission processes can only be performed through numerical calculations due to the wide range of physical conditions present in such tori and their rapid time variation, of the order of tens of milliseconds. We only present here some estimates based on the physical parameters that were employed in the figures of [56]. The dominant neutrino cooling process in these models of accretion tori is electron capture on protons [57], i.e., $e^{-}+p \rightarrow n+\nu_{e}$, which in the degenerate electron limit, has a total emissivity

$$
Q_{\text {Capt }}^{\text {Deg }}=4.2 \times 10^{31} \mu_{10}^{9} \mathrm{erg} \mathrm{cm}^{-3} \mathrm{~s}^{-1},
$$

while eqn.(23) gives

$$
Q_{\mathrm{ee}-\mathrm{Br}}=4.6 \times 10^{30} T_{11}^{7} \mu_{10}^{2} \tilde{N}(\alpha) \mathrm{erg} \mathrm{cm}^{-3} \mathrm{~s}^{-1},
$$

showing that neutrino bremsstrahlung from e-e scattering can make a significant contribution in the "low" density and temperature regions of the torus, and even become the dominant emission mechanism. Maybe more important than energy loss is the production of antineutrinos since it is often considered that the jet of the GRB is triggered by pair production from $\nu-\bar{\nu}$ annihilation along the black-hole rotation axis. Neutrinos are copiously produced by electron capture but antineutrinos come essentially from the outer part of the torus, where $T \sim 10^{10} \mathrm{~K}$ and $\rho \leq 10^{10}$ $\mathrm{g} \mathrm{cm}^{-3}$, and are produced by bremmsstrahlung in nucleon-nucleon collisions, i.e., $N+N^{\prime} \rightarrow N+N^{\prime}+\nu+\bar{\nu}$, with a rate $[58]$

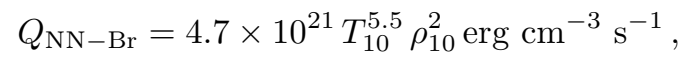

where $\rho_{10}$ is the density in $10^{10} \mathrm{~g} / \mathrm{cc}$. Other important contributions arise from the outermost regions, at densities $\rho \ll$ $10^{10} \mathrm{~g} \mathrm{~cm}^{-3}$ where electrons are non-degenerate and positrons are also present, from the process of pair annihilation, $e^{-}+e^{+} \rightarrow \nu+\bar{\nu}$, with a rate

$$
Q_{\text {Pair }}=4.8 \times 10^{24} T_{10}^{9} \mathrm{erg}_{\mathrm{cm}^{-3} \mathrm{~s}^{-1},}
$$

and positron capture on neutrons, $n+e^{+} \rightarrow p+\bar{\nu}_{e}$, with a rate

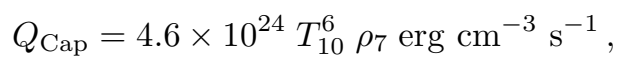

However, neutrinos from e-e scattering which, in contradistinction to processes involving positrons, come from within the whole torus volume, can be expected to make by far the dominant contribution to antineutrino production. Inclusion of this process in future numerical simulations will allow an assessment of its importance.

\footnotetext{
${ }^{6}$ The quark direct urca process $d \rightarrow u+e^{-}+\bar{\nu}_{e}$ would be suppressed due to the mismatch $\delta \mu=\mu_{d}-\mu_{u}$ being large in this phase.
} 

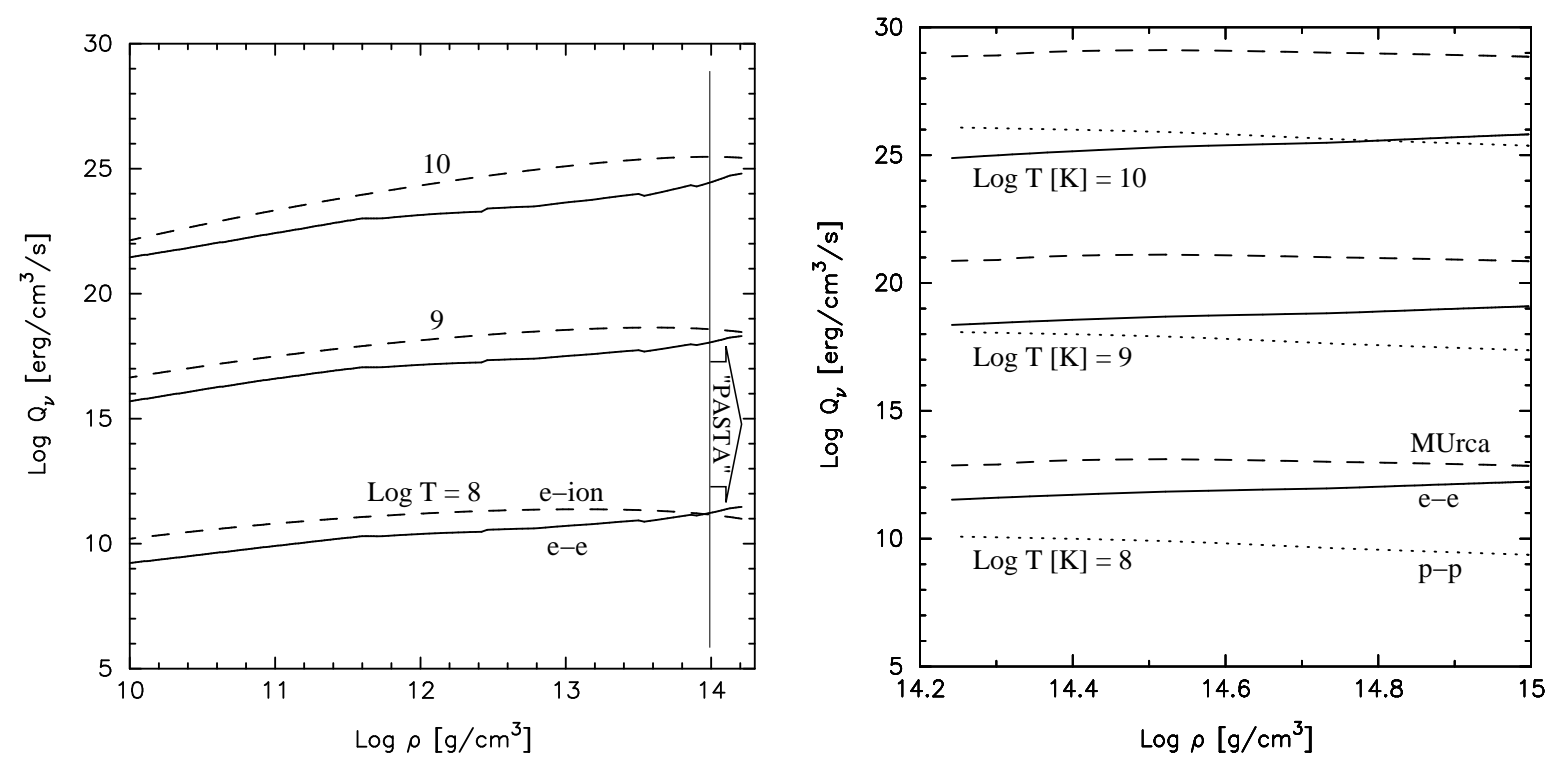

FIG. 5. Relevance of the e-e bremsstrahlung process in neutron stars. Left panel: comparison with the electron-ion bremsstrahlung neutrino emission in the crust, at three typical temperatures. Right panel: comparison with the modified Urca ("MUrca"), sum of the neutron and proton branches, and the proton-proton bremsstrahlung process in the core at the same temperature.

\section{COMPARISON TO NEUTRINO RATES IN NEUTRON STARS}

Neutrino pair bremsstrahlung in electron-electron collisions can also be important in neutron stars and we discuss this in the present section. In the star's crust, the major neutrino emission processes are pair bremsstrahlung processes for electron-ion scattering while in the neutron ${ }^{1} \mathrm{~S}_{0}$ superfluid regime, it is neutrino pair emission by breaking and formation of Cooper pairs at a temperature just below $T_{c}$, the critical temperature for pairing. The Cooper pair process is by far the dominant one but only acts efficiently in a narrow density region where the temperature $T \sim 0.2-0.9 T_{c}$, because of the density dependence of $T_{c}$, while the e-ion and e-e bremsstrahlung processes act in the whole crust. We plot in the left panel of Fig. 5 these last two processes: the emissivity of neutrinos in e-e collisions can dominate only at temperatures $\leq 10^{8} \mathrm{~K}$ and the highest density regime where the "pasta" phase is present. One must emphasize that this "pasta" region actually comprises almost half of the mass of the crust and, moreover, neutrino bremsstrahlung in e-ion collisions is expected to be actually much weaker than shown in the figure [59], by up to two orders of magnitude, due to the lower dimensionality of the nuclei (which are rods and plates instead of spheres).

In the core of the neutron star, the modified Urca and nucleon bremsstrahlung processes are usually considered as the main neutrino emission processes in the absence of any enhanced neutrino emission process such as, e.g., the direct Urca. We plot in the right panel of Fig. 5 the emissivities of the modified Urca process as well as the bremsstrahlung rates in p-p (with rates taken from [60]) and e-e collisions: naturally, the modified Urca process is the dominant one. However, in regions where neutrons are superfluid and $T \ll T_{c}$ the modified Urca process is strongly suppressed, as is neutrino pair emission by the Cooper pair formation process and n-n and n-p associated bremsstrahlung: in such a case only the bremsstrahlung from p-p and e-e scatterings is effective and the latter one is dominant. Notice that in case of proton pairing, the bremsstrahlung rate from p-p scattering will become suppressed but that from e-e will also be significantly reduced because in a superconductor static magnetic fields are screened by the Meissner effect. Photons acquire a Meissner mass and electromagnetic interactions are thus fully screened instead of having their transverse part only Landau-damped. In such a case the calculation of [8] should be applicable for the bremsstrahlung emissivity from e-e scattering, since these authors assumed screening of the whole propagator by a Debye mass $m_{D}$ (instead of $m_{D}$ and $\left.m_{M}\right)$. Again, this process turns out to be the dominant neutrino emission process, although quite inefficient. In the outer part of the core, protons are certainly paired with a $T_{c}$ of the order of $3 \times 10^{9} \mathrm{~K}$ at most [61], while they are likely to be in a normal state at higher densities; the possibility that neutrons may pair at these densities is still very controversial and predictions range from possibly quite high values of $T_{c}$ [62] to almost zero [63]. 


\section{CONCLUSIONS AND OUTLOOK}

We have performed an explicit computation of the neutrino emissivity from neutrino bremsstrahlung in electronelectron collisions in a relativistic degenerate plasma within an astrophysical context. This process has been studied before in the context of neutrino cooling from neutron star crusts, and in gapped superfluids. In this work, we have reinvestigated this process in different astrophysical settings, with closer attention to the dynamics of the screened photon propagator that mediates electron-electron collisions. Compared to previous results [8], we find an enhancement when Landau damping in the transverse part of the photon propagator is taken into account. If we assume simple screening in both the longitudinal and transverse channels of photon exchange, we recover the results of [8]. Our principal results are eqn.(23) and the comparisons in Fig.3, Fig.4 and Fig.5. The inclusion of Landau damping implies a $\mu_{e}^{2} T^{7}$ dependence, instead of the $\mu_{e} T^{8}$ dependence as expected from screening arguments. This leads to an enhancement in the neutrino rate which increases with decreasing temperature, suggesting that e-e bremstrahlung is indeed an efficient cooling process in a certain window of temperature and density. This conclusion could be of particular importance to cooling of strange stars when the interior of the strange star is in a color superconducting phase, such as the CFL phase, which has only gapped quark excitations. The neutrino emission from the interior is then suppressed by several orders of magnitude as the temperature falls below the critical temperature for pairing $\left(T_{c} \sim 50 \mathrm{MeV}\right)$. The overall cooling of the star can be affected due to surface effects as shown in [64,65]. Inclusion of the enhanced neutrino rate in the computations of [64] would be a natural extension of this work, and taken in conjunction with the photon cooling from the strange star surface (which can also influence the light curve at early times) serve to present a more complete picture of strange star cooling.

The quark matter underlying the strange star surface can exist in any one of many phases, and neutrino emission rates have been computed in only a few of them. Of particular interest are the gapless and crystalline phases, which will lead to temperature dependences that are modified from the usual forms in ungapped quark matter. These phases also have unique dispersion relations for certain quark quasiparticles, and consequently, a specific heat per unit volume that is also different from ungapped quark matter. These two factors imply a change in the stellar cooling curve that can be confronted by observations. While we have focused on computing a particular process, our ultimate goal in this direction is to provide more accurate neutrino rates, including the possibility of various quark phases, in order to make accurate predictions for the cooling history of a strange star. This task is complicated by the appearance of non-Fermi liquid behaviour, and the breakdown of a perturbative treatment at temperatures relevant to the long-term cooling of quark matter in the stellar interior.

Finally, we have established the relevance of neutrino bremsstrahlung in e-e collisions for black-hole accretion tori and crustal layers of ordinary neutron stars. The GRB jet may be triggered by $\nu \bar{\nu}$ annihilation, while its subsequent cooling also depends on the neutrino flux, and the e-e process is important for both. Neutron star cooling can also be affected by this process at low temperatures in the pasta-phase of the crust or when neutrons are superfluid in the core. Our results thus find wide-ranging applications for neutron stars, GRB's and strange stars. Increasing data on neutron star cooling, GRB afterglow signatures and theoretical advances on observable signatures of strange stars [66] all suggest that a renewed effort to pin down neutrino rates for astrophysics with improved accuracy is both timely and interesting.

\section{ACKNOWLEDGMENTS}

P.J. is supported by the Department of Energy, Office of Nuclear Physics, contract no. W-31-109-ENG-38. He thanks Guy Moore and Thomas Schäfer for clarifying discussions. The work of D.P. is partially supported by grants from UNAM-DGAPA (PAPIIT-IN112502) and Conacyt (36632-E). C.G. is supported in part by the Natural Sciences and Engineering Research Council of Canada and in part by the Fonds Nature and Technologies of Quebec.

\section{APPENDIX A: $K$-INTEGRATION}

In eqn.(18), a change of variable to $x=\omega / k$ gives

$$
\begin{aligned}
I_{k} & =\frac{1}{\omega^{3}} \int_{a}^{\sim \omega / l_{1}} d x \frac{x^{2}}{\left[\left(1-x^{2}\right)^{2}+b^{2} x^{6}\right]}\left[1-\left\{\frac{a\left(1-x^{2}\right)+x^{2}}{x}\right\}^{2}\right] \\
a & =\frac{\omega}{2 \mu_{e}} ; \quad b=\frac{\pi}{4}\left(\frac{m_{D}}{\omega}\right)^{2} .
\end{aligned}
$$


For the physical conditions of interest, $a \ll 1$ and $b \gg 1$, which enables us to use a much simpler form

$$
I_{k}=\frac{1}{\omega^{3}} \int_{a}^{\sim \omega / l_{1}} d x \frac{x^{2}}{\left[1+b^{2} x^{6}\right]}
$$

The integrand is not very different than in eqn.(A1) for the entire range of integration (note that $l_{1} \sim T$ so that $\omega / l_{1} \sim 1$ ), as evident from Fig. 6 below.

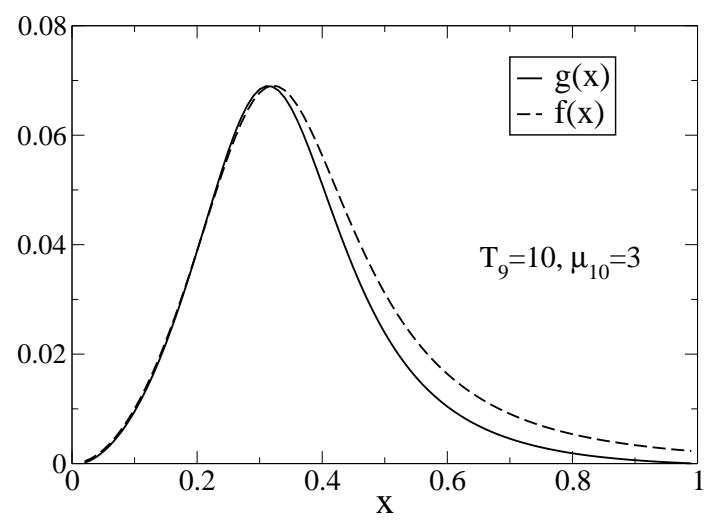

FIG. 6. The integrands from eqn.(A1) and eqn.(A3) labeled $g(x)$ and $f(x)$ respectively. The temperature is $T_{9}=10$ $\left(T=10^{10} \mathrm{~K}\right)$ and $\mu_{10}=3\left(\mu_{e}=30 \mathrm{MeV}\right)$.

The integral in eqn.(A3) is straightforward and yields

$$
I_{k} \approx \frac{4}{3 \pi m_{D}^{2} \omega}\left[\frac{\pi}{2}-\tan ^{-1}\left(\frac{l_{1}^{3}}{\frac{\pi}{4} m_{D}^{2} \omega}\right)\right] .
$$

\section{APPENDIX B: NUMERICAL EVALUATION OF $N(\alpha)$}

The integral $I(\bar{\Omega})$ in eqn. $(21)$ is

$$
\begin{aligned}
I(\bar{\Omega}) & =\int_{0}^{\infty} d \bar{\omega} \frac{(\bar{\omega}+\bar{\Omega})}{\sinh \bar{\omega} \sinh (\bar{\omega}+\bar{\Omega})}\left[\frac{\pi}{2}-\tan ^{-1}\left(\frac{4}{\pi} \frac{(\bar{\omega}+\bar{\Omega})^{3}}{\bar{\omega} \alpha^{2}}\right)\right]+\int_{0}^{\bar{\Omega}} d \bar{\omega} \frac{\omega}{\sinh \bar{\omega} \sinh (\bar{\omega}+\bar{\Omega})}\left[\frac{\pi}{2}-\tan ^{-1}\left(\frac{4}{\pi} \frac{(\bar{\omega}+\bar{\Omega})^{2}}{\alpha^{2}}\right)\right] \\
& +\int_{0}^{\bar{\Omega}} d \bar{\omega} \frac{(\bar{\Omega}-\bar{\omega})}{\sinh \bar{\omega} \sinh (\bar{\Omega}-\bar{\omega})}\left[\frac{\pi}{2}-\tan ^{-1}\left(\frac{4}{\pi} \frac{(\bar{\Omega}-\bar{\omega})^{3}}{\bar{\omega} \alpha^{2}}\right)\right]+\int_{2 \bar{\Omega}}^{\infty} d \bar{\omega} \frac{(\bar{\Omega}-\bar{\omega})}{\sinh \bar{\omega} \sinh (\bar{\Omega}-\bar{\omega})}\left[\frac{\pi}{2}-\tan ^{-1}\left(\frac{4}{\pi} \frac{(\bar{\omega}-\bar{\Omega})^{3}}{\bar{\omega} \alpha^{2}}\right)\right]
\end{aligned}
$$

with

$$
\bar{\omega}=\frac{\omega}{2 T}
$$

This integral is well-behaved for all finite values of $\bar{\Omega}$, even as $\bar{\omega} \rightarrow 0$. At $\bar{\Omega}=0$, the integrand diverges at $\bar{\omega}=0$, but the overall emissivity is clearly zero. Thus, a numerical evaluation of $N(\alpha)$ from eqn.(21) with the above expression for $I(\Omega)$ is feasible. The rapid exponential fall-off of the integrand with respect to $\bar{\omega}$ and $\bar{\Omega}$ implies a rapid convergence and high degree of accuracy in the final result. The integral $N(\alpha)$ is evaluated by Gauss-Legendre quadratures and the normalized $\tilde{N}$ that appears in eqn. (23), is plotted in Fig.2. 
[1] G. G. Raffelt, Stars as Laboratories for Fundamental Physics, (C) The University of Chicago Press, 1996.

[2] D. G. Yakovlev and C. J. Pethick, Ann. Rev. Astron. Astrophys. 42, 169 (2004).

[3] D. Page, U. Geppert and F. Weber, to be published in Nucl. Phys. A. (astro-ph/0508056).

[4] J. M. Lattimer, C. J. Pethick, M. Prakash, and P. Haensel, Phys. Rev. Lett. 66, 2701 (1991).

[5] M. Prakash, M. Prakash, J. M. Lattimer and C. J. Pethick, ApJ 390, 77 (1992).

[6] N. Iwamoto, Phys. Rev. Lett. 44, 1640 (1980); A. Burrows, ibid 1637.

[7] D. G. Yakovlev, A. D. Kaminker, O. Y. Gnedin and P. Haensel, Phys. Rep. 351, 1 (2001).

[8] A. D. Kaminker and P. Haensel, Acta Phys. Polon. B30, 1125 (1999).

[9] G. Baym, H. Monien, C. J. Pethick, and D. G. Ravenhall, Phys. Rev. Lett. 64, 1867 (1990).

[10] H. Heiselberg and C. J. Pethick, Phys. Rev. D48, 2916 (1993).

[11] L. B. Leinson, Phys. Lett. B469, 166 (2000).

[12] J. I. Kapusta, Finite-Temperature Field Theory, (c) Cambridge University Press, 1989.

[13] T. Schäfer and K. Schwenzer, Phys. Rev. D70, 054007 (2004).

[14] T. Schäfer and K. Schwenzer, Phys. Rev. D70, 114037 (2004).

[15] F. E. Low, Phys. Rev. 110, 974 (1958).

[16] P. Cazzola and A. Saggion, Nuovo Cimento A63, 354 (1969); P. Cazzola and A. Saggion, ibid 367 (1969).

[17] E. Braaten and D. Segel, Phys. Rev. D48, 1478 (1993).

[18] S. Ratkovic, S. I. Dutta and M. Prakash, Phys. Rev. C67, 123002 (2003).

[19] S. I. Dutta, S. Ratkovic and M. Prakash, Phys. Rev. D69, 023005 (2004).

[20] L. B. Leinson and M. Perez, Nucl. Phys. B597, 279 (2001).

[21] E. Farhi and R. L. Jaffe, Phys. Rev. D30, 2379 (1984).

[22] C. Alcock, E. Farhi and A. V. Olinto, Astrophys. J. 310, 261 (1986).

[23] P. Jaikumar, S. Reddy and A. Steiner, nucl-th/0507055.

[24] M. Alford, K. Rajagopal and F. Wilczek, Nucl. Phys. B537, 443 (1999).

[25] J. Madsen, Phys. Rev. Lett. 87, 172003 (2003); V. V. Usov, Phys. Rev. D70, 067301 (2004).

[26] I. A. Shovkovy and P. J. Ellis, Phys. Rev. C66, 015802 (2002).

[27] P. Jaikumar, M. Prakash and T. Schäfer, Phys. Rev. D66, 063003 (2002).

[28] N. Iwamoto, Annals. Phys. 141, 1 (1982).

[29] S. Reddy, M. Sadzikowski and M. Tachibana, Nucl. Phys. A714, 337 (2003).

[30] P. F. Bedaque and T. Schäfer, Nucl. Phys. A 697, 802 (2002).

[31] T. Schäfer, Phys. Rev. Lett 85, 5531 (2000).

[32] P. F. Bedaque, Phys. Lett. B524, 137 (2002).

[33] M.Alford, C. Kouvaris and K. Rajagopal, Phys. Rev. Lett. 92, 222001 (2004).

[34] M.Alford, C. Kouvaris and K. Rajagopal, Phys. Rev. D71, 054009 (2004).

[35] I. A. Shovkovy and M. Huang, Phys. Lett. B564, 205 (2003).

[36] M. Alford, P. Jotwani, C. Kouvaris, J. Kundu and K. Rajagopal, astro-ph/0411560.

[37] A. Schmitt, Phys. Rev. D71, 054016 (2005); also see author's PhD Thesis: nucl-th/0405076.

[38] M. Huang and I. A. Shovkovy, Nucl. Phys. A 729, 835 (2003).

[39] M. Alford, C. Kouvaris and K. Rajagopal, in Proceedings of Strong and Electroweak Matter 2004.

[40] R. Casalbuoni and G. Nardulli, Rev. Mod. Phys. 76, 263 (2004).

[41] J. A. Bowers and K. Rajagopal, Phys. Rev. D65, 094022 (2002).

[42] R. Casalbuoni, R. Gatto and G. Nardulli, Phys. Lett. B543, 139 (2002).

[43] C. Price, Phys. Rev. D22, 1910 (1982).

[44] R. C. Duncan, S. L. Shapiro and I. Wasserman, ApJ 267, 338 (1983).

[45] P. Jaikumar and M. Prakash, Phys. Lett. B516, 345 (2001).

[46] D. H. Rischke, Phys. Rev. D62, 054017 (2000).

[47] M. Alford and K. Rajagopal, JHEP 0206, 31 (2002).

[48] M. Huang and I. A. Shovkovy, Phys. Rev. D70, 094030 (2004).

[49] R. Casalbuoni, R. Gatto, M. Mannarelli, G. Nardulli and M. Ruggieri, Phys. Lett. B605, 362 (2005).

[50] M. Alford and Q. Wang, J. Phys. G31, 719 (2005).

[51] B. Y. Park, M. Rho, A. Wirzba and I. Zahed, Phys. Rev. D62, 034015 (2000).

[52] P. Jaikumar and I. Zahed, Phys. Rev. D64, 014035 (2001).

[53] R. Casalbuoni, E. Fabiano, R. Gatto, M. Mannarelli and G. Nardulli, Phys. Rev. D66, 094006 (2002).

[54] S. E. Woosley, Astrophys. J. 405, 273 (1993).

[55] W. Lee, E. Ramirez-Ruiz and D. Page, Astrophys. J. 608, L5 (2004). 
[56] W. Lee, E. Ramirez-Ruiz and D. Page, Astrophys. J., in press (2005).

[57] K. Kohri and S. Mineshige, Astrophys. J. 577, 311 (2002).

[58] S. Hannestad and G. Raffelt, Astrophys. J. 507, 339 (1998).

[59] A. D. Kaminker, C. J. Pethick, A. Y. Potekhin, V. Thorsson, and D. G. Yakovlev, Astron. \& Astrophys. 343, 1009 (1999).

[60] D. G. Yakovlev, and K. P. Levenfish, Astron. \& Astrophys. 297, 717 (1995).

[61] J. Wambach, T. L. Ainsworth, and D. Pines, in Neutron Stars: Theory versus Observations, J. Ventura and D. Pines (eds), Kluwer, 1991, p. 37.

[62] M. Baldo, Ø. Elgarøy, L. Engvik, M. Hjorth-Jensen, and H.-J. Schulze, Phys. Rev. C58, 1921 (1998).

[63] A. Schwenk and B. Friman, Phys. Rev. Lett. 92, 082501 (2004).

[64] D. Page and V. V. Usov, Phys. Rev. Lett. 89, 131101 (2002).

[65] P. Jaikumar, C. Gale, D. Page and M. Prakash, Phys. Rev. D70, 023004 (2004).

[66] D. Page and A. Cumming, submitted to Astrophys. J. Lett. (2005) (astro-ph/0508444). 